\title{
Effectiveness of Interventions for Children With Developmental Coordination Disorder in Physical Therapy Contexts: A Systematic Literature Review and Meta-Analysis
}

\author{
Nkechi Offor, Peace Ossom Williamson, and Priscila Caçola
} University of Texas-Arlington

\begin{abstract}
Purpose: The purpose of this systematic literature review and meta-analysis is to identify the types of interventions in physical therapy contexts that have been explored in children with developmental coordination disorder, the most common variables being addressed, and whether these interventions are effective. Method: This systematic search of MEDLINE, PEDro, CINAHL, SPORTDiscus, PsycINFO, and Cochrane Library identified interventions in physical therapy contexts for children and adolescents with DCD, and studies were screened using these criteria and assessed using the PEDro and NIH quality assessment scales. AMSTAR was used to evaluate systematic reviews and a meta-analysis was conducted. Results: From the articles reviewed, 29 articles of moderate to good quality were included in the qualitative analysis. Task-oriented approaches as well as motor skill training-based interventions have shown beneficial effects in improving motor function in children with DCD. Data from 14 articles was extracted for inclusion in the meta-analysis, providing support for the effectiveness of physical therapy interventions against inaction. Conclusion: Researchers recommend the use of task-oriented and traditional physical therapy interventions for children with DCD. In addition, interventions in physical therapy contexts need clear goals and outcome measures for individual children.
\end{abstract}

Keywords: developmental coordination disorder, child, adolescent, physical therapy, interventions, systematic reviews, meta-analysis, motor skill disorders

Developmental coordination disorder (DCD) is a condition in which difficulties in motor skills exist but are not due to the overall intellect, primary sensor, or motor neurology of the individual. A primary characteristic of DCD is the difficulty in learning and performing everyday tasks in all aspects of life (home, school, 
play, etc.). The estimated prevalence of DCD ranges between $2 \%$ and $7 \%$ of all school-aged children (American Psychiatric Association [APA], 2013) with some reports suggesting that boys have a higher incidence than girls (Hillier, 2007). Children with DCD generally perform more poorly than other children on a variety of measures reflective of motor control and motor learning as well as sensory and perceptual processing. DCD can occur with other conditions such as specific language impairment (SLI) or attention-deficit hyperactivity disorder (ADHD) (Cermark, Gubbay, \& Larkin, 2009).

According to the Diagnostic and Statistical Manual of Mental Disorders (5th ed.; DSM-5; APA, 2013), the criteria for a DCD diagnosis involve the following characteristics:

(a) Acquisition and execution of coordinated motor skills are below what would be expected at a given chronologic age and opportunity for skill learning and use; difficulties are manifested as clumsiness (e.g. dropping or bumping into objects) and as slowness and inaccuracy of performance of motor skills (e.g. catching an object, using scissors, handwriting, riding a bike, or participating in sports), (b) The motor skills deficit significantly or persistently interferes with activities of daily living appropriate to the chronologic age (e.g. self-care and self-maintenance) and impacts academic/school productivity, prevocational and vocational activities, leisure, and play, (c) Onset of symptoms is in the early developmental period, (d) The motor skills deficits cannot be better explained by intellectual disability or visual impairment and are not attributable to a neurologic condition affecting movement (e.g. cerebral palsy, muscular dystrophy, or a degenerative disorder). (p. 74)

While there is no cure for this condition, researchers have established that children with DCD benefit from some form of motor intervention (Smits-Engelsman et al., 2013). Various intervention programs have been implemented to help improve motor abilities in children with DCD, ranging from cognitive orientation to occupational performance (CO-OP; Miller, Polatajko, Missiuna, Mandich, \& Macnab, 2001) to chemical supplementation (Smits-Engelsman et al., 2013).

According to the literature, several impairments of children with DCD can be improved through interventions performed in physical therapy contexts, such as core stability programs, muscle strength training programs, and balance training programs (Au et al., 2014; Kaufman \& Schilling, 2007; Silkwood-Sherer, Killian, Long, \& Martin, 2012). Physical therapy interventions refer to programs being established by the therapist to improve the variables (outcome measures) being analyzed. According to the American Physical Therapy Association (2015), physical therapy is a health care profession in which licensed professionals use treatment techniques and fitness- and wellness-oriented programs to reduce an individual's pain, restore function and movement, and to prevent disability or loss of mobility. More specifically for children with DCD, physical therapists can work to improve coordination difficulties (considered the primary impairment in DCD) and also to prevent the development of activity limitations or participation restrictions (considered as secondary consequences) that result from an impairment or impairments in body structure or function (Camden, Rivard, Pollock, \& Missiuna, 2012).

Physical therapists can also work to improve mobility and function of various body parts to further develop the motor proficiency of children with DCD. For 
example, a therapist may work on core or limb/shoulder girdle stability to improve motor skills associated with the upper or lower body, such as throwing, catching, kicking, or balance. A recent study compared a task-oriented motor training with a core stability program in improving motor proficiency in children with DCD, and significant improvements were seen in motor proficiency in both groups ( $\mathrm{Au}$ et al., 2014). Interventions in physical therapy contexts range in strategy, and this systematic literature review groups these interventions in three categories.

One of the categories is traditional physical therapy, which refers to common treatment modalities used by therapists such as strength or balance exercises, or noninvasive treatments such as an ultrasound or electrical stimulation. Strength training, also known as resistance training, is an example of traditional physical therapy involving exercises that use the resistance of body weight, resistance bands, or weight machines, to induce muscular contractions resulting in improved muscular strength, power, size, and endurance. Another traditional physical therapy intervention method is core stability training, which develops the muscles of the lumbopelvic and abdominal regions that provide stability of the spine during movement and during a change in posture (Bhayani \& Singaravelan, 2012). The purpose of core stability training is to improve strength, balance, and coordination (Kane \& Bell, 2009) along with extremity function, proximal stability, and endurance (Bhayani \& Singaravelan, 2012).

The second category is contemporary physical therapy, or novel treatments used by physical therapists, which includes active virtual gaming, hippotherapy, and interactive metronome training. Other contemporary physical therapy methods can be combined. For example, aquatic therapy involves water modality incorporated into an exercise program; and rebound training involves the use of trampolines to improve balance and stability. Active virtual reality gaming (AVG) incorporates a technological component as the therapy element. AVG has been shown to effectively improve balance in children with DCD as well as other aspects of gross motor skill development (Gonsalves, Campbell, Jensen, \& Straker, 2015) and is being used by physical therapists as a treatment option to improve balance as well as muscle strength. Rebound therapy is a type of contemporary physical therapy intervention that uses a trampoline as a treatment modality. The use of a trampoline has a unique effect on the body by stimulating the sympathetic and vestibular systems and the proprioceptors, consequently developing muscle tone (Addy, 1996). Hippotherapy differs from the others in that the means of treatment involves the movement pattern of a horse, and it is used to achieve effective results on balance, postural control, and other motor skills.

The third category includes treatments involving task-oriented training. Task-oriented treatment approaches tend to concentrate on improving motor skills by learning them while working on the particular task that causes the child difficulty (Smits-Engelsman et al., 2013). Physical therapists from the Netherlands developed a type of task-oriented training called neuromotor task training (NTT). NTT is based on a cognitive neuroscience approach to motor control (Niemeijer, Smits-Engelsman, \& Schoemaker, 2007; Schoemaker, Niemeijer, Reynders, \& Smits-Engelsman, 2003), and uses a combination of motor learning and ecological principle of skill development through repeated learning sessions, as well as task and environmental constraint manipulations (Smits-Engelsman et al., 2013). A recent systematic literature review on the efficacy of motor interventions for children 
with DCD (task-oriented training, traditional physical and occupational therapy, process-oriented therapies, and chemical supplements) found evidence supporting traditional physical and occupational therapy and task-oriented interventions (Smits-Engelsman et al., 2013). In this review, both task-oriented training interventions, including NTT, and motor training-based interventions, including physical and occupational therapy, showed strong treatment effects. Smits-Engelsman and colleagues (2013) also concluded that therapies should include task-oriented elements to promote transfer.

The systematic review process involves gathering all existing knowledge using a thorough and methodological approach, as well as summarizing the best available research on a specific topic or question to provide a repeatable method that reduces reporting bias. Because of existing evidence for interventions in physical therapy contexts in relation to motor proficiency of children with DCD, the purpose of this study is to systematically conduct a review gathering all existing knowledge on these interventions in this population. More specifically, the goals of this study are to identify the types of interventions in physical therapy contexts that have been explored in children with DCD and the most common variables being addressed (such as balance, motor coordination and timing, muscle strength and function, etc.), and to apply a meta-analysis to verify whether these interventions are effective.

\section{Method}

\section{Search Strategy for Identification of Studies}

A specific search strategy was created and developed to identify a comprehensive collection of research. This strategy was then peer reviewed by a medical librarian with extensive experience in searching due to publishing systematic reviews and serving on a medical institutional review board. The strategy was adapted and used to search MEDLINE (via PubMed, 1946-), PEDro (1929-), CINAHL (via EBSCO, 1937-), SPORTDiscus (via EBSCO, 1985-), PsycINFO (via EBSCO, 1600-), and Cochrane Library (via Wiley, 1992-) to retrieve articles published until March 2015. The keywords and subject headings were grouped by three topics-DCD, children, and physical therapy intervention. Each of the terms within these topics were combined using the OR Boolean operator, then the three groups were combined with the AND Boolean operator. The list of keywords and their search method is in Table 1. To restrict articles, exclusionary terms including cerebral palsy, stroke, traumatic brain injury, leukodystrophy, and Krabbe's disease followed the "NOT" Boolean operator in the search. English Only filters were used. Supplemental searches were applied in WorldCat (via FirstSearch) and ProQuest Dissertations and Theses (1637-) databases. Searches were conducted between March 1 and 15, 2015, and repeated for the retrieval of new publications on August 14, 2015. Resources were also collected through author name searching and a review of the reference lists from each of the articles that were moved forward from first screening.

\section{Process for Inclusion or Exclusion of Studies}

The total number of studies matching the search terms was 3,570. Each abstract was then reviewed and assessed to determine inclusion or exclusion in the review. 


\section{Table 1 Search Keywords by Group}

\begin{tabular}{|c|c|c|}
\hline $\mathrm{DCD}^{\mathrm{a}}$ & Children & PT interventions ${ }^{a}$ \\
\hline Apraxia* & Adolescen* & Imagery training \\
\hline Clumsiness & Babies & Intervention \\
\hline Clumsy & Baby & Kinesthetic training \\
\hline Congenital maladroitness & Boy & Motor learning \\
\hline DCD & Child* & $\begin{array}{l}\text { Neurodevelopmental } \\
\text { treatment }\end{array}$ \\
\hline Developmental coordination disorder & Elementary school & NTT \\
\hline Disorder of sensory integration & Girl & Perceptual motor training \\
\hline Dys-coordination & High school & Physical therap* \\
\hline Dysprax* & High school & Physiotherap* \\
\hline In-coordination & Infan* & Process-orient $*$ \\
\hline Mild motor problems & Juvenile* & Sensory integration \\
\hline Minimal brain function & Kindergar* & Skill training \\
\hline Minimal cerebral dysfunction & Minor* & Task training \\
\hline Minor neurological disorder & Neonat* & Task-orient* \\
\hline Motor control and perception & Newborn & Task-specific training \\
\hline Motor coordination difficulties/problems & Nursery & Treatment \\
\hline Motor delay & Pediatric* & \\
\hline Motor learning difficulties/problems & Pediatric* & \\
\hline Motor skills disorder & Perinat* & \\
\hline Nonverbal learning & Postnat* & \\
\hline Perceptual motor dysfunction & Prepuberty & \\
\hline Perceptual motor impairment & Preschool & \\
\hline Physic* awkward* & Primary school & \\
\hline Psychomotor disorders & Puberty & \\
\hline Sensorimotor difficulties/dysfunction & Pubescen* & \\
\hline Sensory integrative dysfunction & School age* & \\
\hline \multirow{8}{*}{$\begin{array}{l}\text { Specific developmental disorder of } \\
\text { motor function }\end{array}$} & School age* & \\
\hline & Schoolchild* & \\
\hline & Secondary school & \\
\hline & Teen* & \\
\hline & Toddler* & \\
\hline & Unerag* & \\
\hline & Year* old* & \\
\hline & Youth* & \\
\hline
\end{tabular}

Note. The asterisk was used for truncation searching.

${ }^{a}$ Quotation marks were used as needed for phrases. 
The inclusion criteria were as follows: (a) literature is written in English, (b) participants are under 18 years of age, (c) participants are diagnosed with DCD, and (d) a physical therapy intervention is tested in the study. Studies that did not explicitly test interventions were excluded. Two reviewers used these criteria again when independently screening the full text of each one of the 109 resources that had not been excluded by the first screening.

\section{Quality Assessment and Selection of Studies}

Thirty-four studies met the inclusion criteria, and these remaining studies were evaluated for quality in methodology. The Physiotherapy Evidence Database's PEDro scale (1999) was used to evaluate controlled trials, AMSTAR was used to evaluate systematic reviews (Shea et al., 2007), and the National Institutes of Health (NIH) quality assessment scales (2014) were used to evaluate the other studies. Two independent raters scored each study, and studies that received a poor rating from either rater were reviewed by a third expert. The studies that received a poor rating from the third reviewer were then excluded from the systematic review.

\section{Data Extraction and Synthesis}

One author extracted data for the meta-analysis, including study types, participant characteristics, group size, intervention characteristics, and outcome characteristics, while another reviewer checked the extracted data. The resources that reported either the means and standard deviations or the mean difference and effect size of motor proficiency composite scores were included in this process. Because motor proficiency was assessed using a range of instruments, the standardized mean difference (SMD) with $95 \%$ confidence intervals (CI) was reported. The Review Manager (RevMan 5) software by the Cochrane Collaboration was used for the meta-analysis. Because many studies involved nonrandomized intervention or control groups, an overall estimate of effect was sought via inverse variance outcome assessment. Results are reported as MD (95\% confidence interval), and the random effects model was used because of the heterogeneity among interventions. For studies using various outcome measures, the data reported via the Movement Assessment Battery for Children was included. For studies in which lower outcome scores represented improvement, the mean change was reversed for consistency with the other data. The process of inclusion and exclusion can be seen in Figure 1.

\section{RESULTS}

As a result of the search procedure described above, a total of 29 studies met the criteria for inclusion (full description of the studies is provided in Table A1, in the appendix) in the systematic review qualitative analysis. Five of these articles used a task-oriented approach, six used contemporary PT, and the remaining 18 used traditional PT. Of these 29 articles, two included occupational therapy (OT) coupled with their physical therapy intervention approach (Addy, 1996; Maldonado, Miller, Moran, \& Eberhart, 2012). Thirteen of the 29 studies from the systematic review were included in the meta-analysis. 


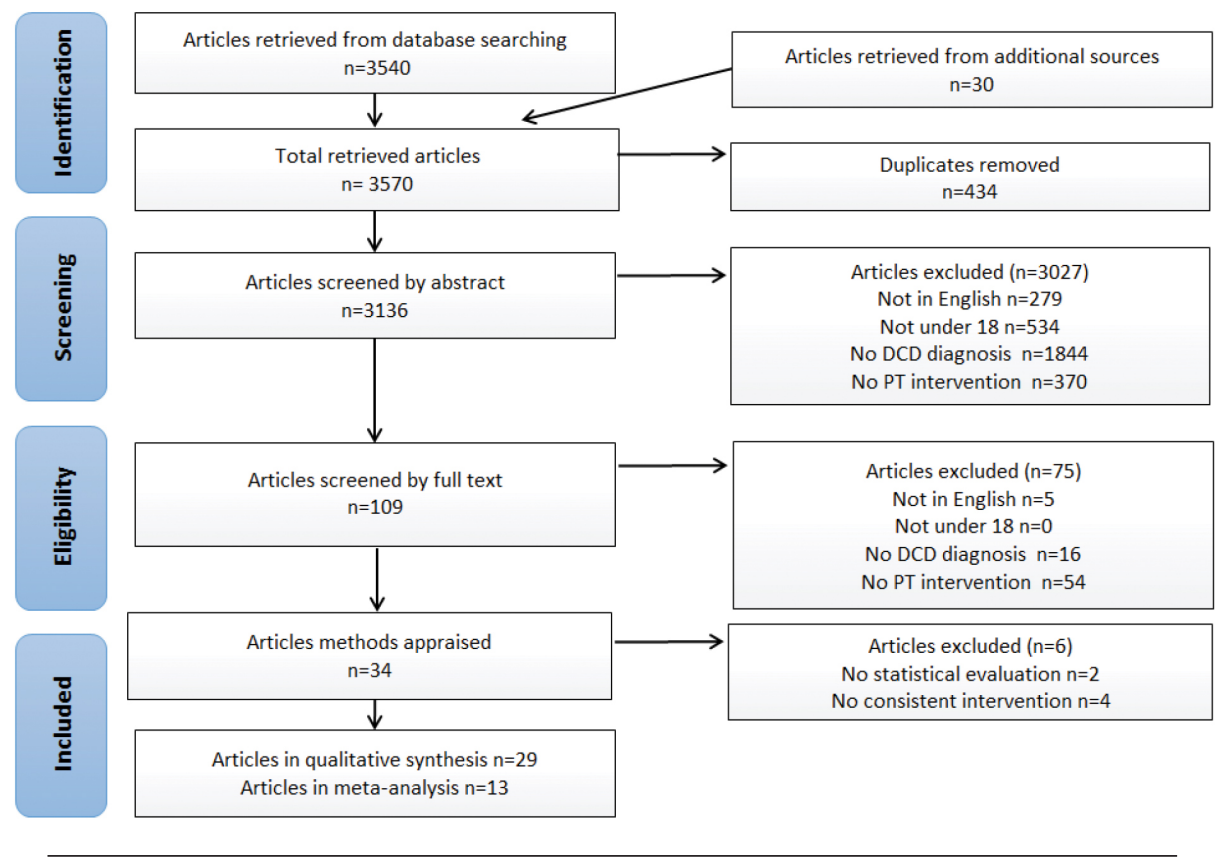

Figure 1 - Flowchart of the inclusion and exclusion process of studies.

In the following section, we describe the statistical methods used and the outcome of those methods. The assessment tools used in all the studies are listed, and the types of interventions in physical therapy contexts are categorized and described in more detail, as well as the results of each study.

\section{Assessment Tests}

The following tests were used to differentiate children with DCD from typically developing children: Movement Assessment Battery for Children, 2nd edition (MABC-2); Bruininks-Oseretsky Test of Motor Proficiency, 2nd edition (BOTMP2); Developmental Coordination Disorder Questionnaire; the Test of Motor Impairment (TOMI); the Body Coordination Test for Children (BCTC); Test of Gross Motor Development, 2nd edition (TGMD-2); Neuro-Sensory Motor Developmental Assessment (NSMDA); the Wechsler Intelligence Scale for Children; the Balance Test; the Kaufman Brief Intelligence Test, 2nd edition (KBIT-2); Vicon; the Diagnostic and Statistical Manual of Mental Disorders; Neurodevelopmental Physiotherapy Assessment.

The following tests were used to evaluate differences between the pre- and posttests: MABC-2, BOT-2, TGMD-2, TOMI, the Sensory Organization Test (SOT), Unilateral Stance Test (UST), Motor Control Test (MCT), Cybex Dynamometer, the Children's Self-Perceptions of Adequacy in Predilection for Physical Activity (CSAPPA), the 5-point Facial Hedonic Scale, the Perceived Motor Competence Scale, the Pictorial Scale of Perceived Competence and Social Acceptance 
(PSPCSA), Proprioceptive Testing, the Pediatric Balance Scale, Activities Scale for Kids Performance (ASKp), the Dysgraphia Scale (BHK), the Harter's Scale of Perceived Competence/Harter and Pike's Pictorial Scale of Perceived Competence, and the Graphomotor Task.

\section{Intervention Characteristics}

As is evidenced in Figure 2, the meta-analysis of motor proficiency improvement in physical therapy interventions was significantly different between intervention and control groups (SMD 8.26, 95\% CI 5.47-11.05, $p<.01$ ) and within intervention groups from baseline (SMD 5.34, 95\% CI 3.43-7.24, $p<.01$ ). Figure 2 presents the diagram representations of the meta-analysis where the mean difference is illustrated in these charts.

Physical therapy interventions included in this review involve traditional, contemporary, and task-oriented methods. Traditional physical therapy had the greatest overall effect size pre- and posttest scores in children with DCD (SMD 7.61, 95\% CI 4.20-11.01) and was applied through perceptual motor training, strength training, and core stability training. Contemporary physical therapy tested in some studies had the second greatest effect size (SMD 4.49, 95\% CI -1.75-10.72). NTT (SMD $3.24,95 \%$ CI 0.24-6.24) was an effective physical therapy intervention present in three of the five task-oriented intervention studies. The findings of the reviewed studies were categorized into the types of physical therapy interventions used and are described below.

\section{Traditional Physical Therapy}

Motor Skills Training. Ten of the 29 studies included in the SLR (including three randomized controlled trials) explored the effectiveness of motor skills training using 248 children ages 6-17 years (Chia \& Chua, 2002; Iversen, Ellertsen, Tytlandsvik, \& Nødland, 2005; Hodges, 2014; Hung \& Pang, 2010; Lee \& Smith, 1998; Maldonado et al., 2012; Morton, 2015; Peters \& Wright, 1999; Schoemaker, Hijlkema, \& Kalverboer, 1994; Watter \& Bullock, 1989). In studies where frequency was reported, physical therapy was provided weekly (Hung \& Pang, 2010; Lee \& Smith, 1998; Morton, 2015; Peters \& Wright, 1999), biweekly, or three times a week (Watter \& Bullock, 1989) for 8-12 weeks. In one study, participants also had a daily home program (Lee \& Smith, 1998). For the ten studies, progress was recorded at the end of the treatment sessions, and for two studies, progress was also recorded at a review 12 weeks to 8 months later (Lee \& Smith, 1998; Morton, 2015). All children in one study were treated by the same therapist to maintain consistency (Schoemaker et al., 1994). In one study, scores taken at the end of the treatment period showed an improvement in 50-90\% with a mean of $72 \%$ per patient (Lee \& Smith, 1998). Scores for motor proficiency revealed a significant interaction between group and treatment in three studies (Chia \& Chua, 2002; Morton, 2015; \& Schoemaker et al., 1994) and an interaction between baseline and postintervention in another study (Peters \& Wright, 1999). Of the ten motor skills studies, six were included in the meta-analysis, and the overall effect of motor skills training on motor proficiency in children with DCD can be seen in Figure 3. 

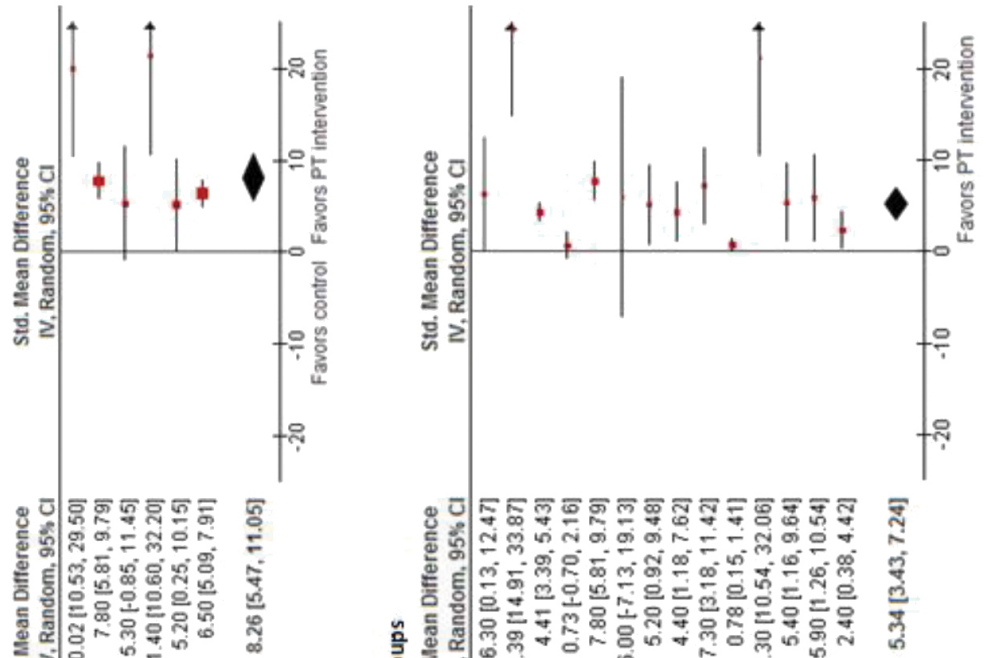

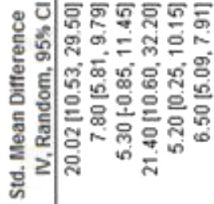

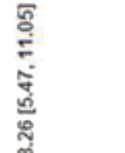

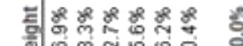

उंक्त

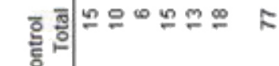

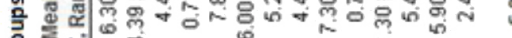

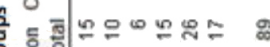

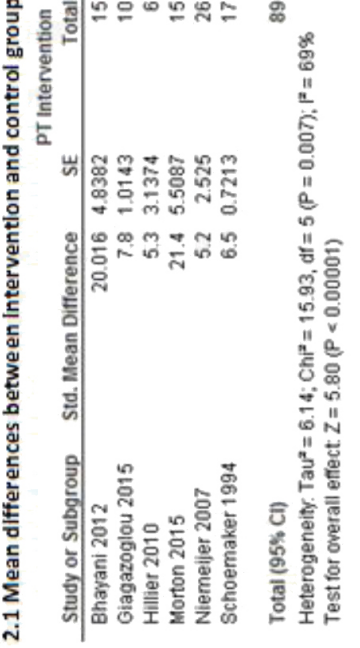

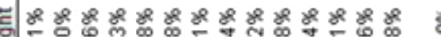

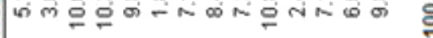

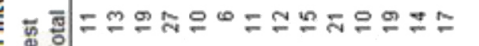

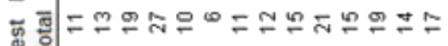

宽

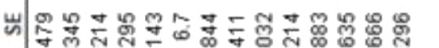

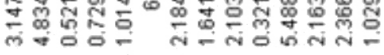

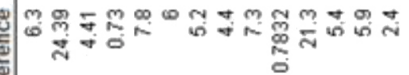




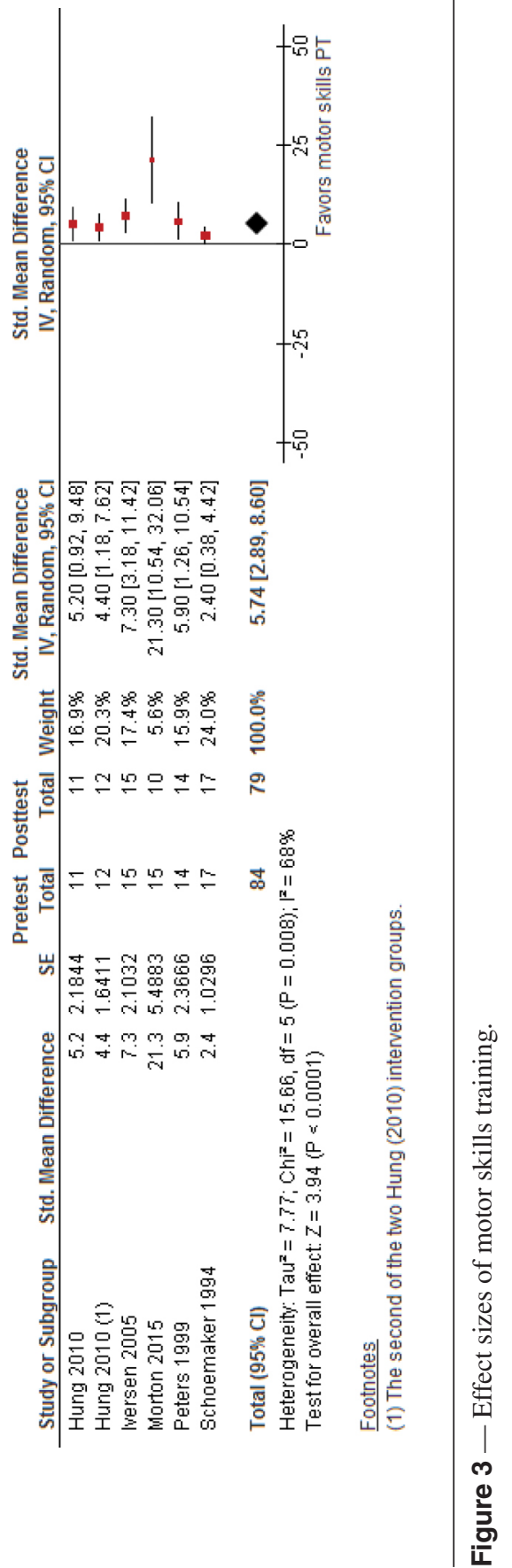


Balance was an outcome measured in several studies (Hodges, 2014). One study noted improvements in the treatment group for academic scores, learning behavior, and social-emotional status; however, these were not statistically significant (Chia \& Chua, 2002). The case study by Hodges (2014) found that after eight months of their physical therapy program, the child improved in balance, coordination, gross motor development, cardiovascular endurance, and self-esteem. In two studies, there were two intervention groups (Hung \& Pang, 2010; Iversen et al., 2005). In one study, one group received group-based training and the other group received individual-based training (Hung \& Pang, 2010). Although the difference in MABC total impairment score was not significant between the two intervention groups ( $p=.38$ ), Hung and Pang (2010) found a significant reduction in scores of the individual-based training intervention group (mean-5.2 (SD 5.1), $p=.01$ ) and the group-based training intervention group (mean-4.4 (SD 5.0), $p<.01$ ).

In the other study, Group A $(N=15)$ received a high-dosage of motor skills intervention with an increase in parental involvement, while Group B $(N=15)$ received a low-dosage with limited parental involvement. After 1-4 years of the intervention, there was a follow-up of parental description of the child's coping at home and school. Even though no significant differences between groups were found with regard to MABC sum scores (Group A mean change $(S D)=13.8(6.66)$, Group B mean change $(S D)=16.0(6.97))$, the parents from Group A reported an overall more favorable situation at the levels of activity and participation.

Strength Training. Strength training is used to improve gross motor function, and three studies provided evidence to support that strength and power may be the underlying contributing factor to motor difficulties (Fong, Chung, Chow, Ma, \& Tsang, 2013; Kaufman \& Schilling, 2007; Menz, Hatten, \& Beuttler, 2011). One randomized trial researched the effect of tae kwon do training (TKD) on knee muscle strength and reactive and static balance control in children with DCD (Fong et al., 2013). After the training, the intervention participants' isokinetic knee muscle strength, at $180 \%$, did not differ significantly from the control group of typically developing (TD) children $(p>.01)$. The UST body sway velocity in the DCD-TKD group was slower at baseline than the control group of children with DCD $(p<.001)$, and sway velocity of that same group postintervention was comparable to that of the TD control group $(p>.05)$. However, no improvement in balance was found in the MCT $(p>.02)$.

A case study performed by Kaufman and Schilling (2007) on a five-year-old child used a strength training program that involved a sequence of large muscle group exercises to small muscle group multijoint and single-joint exercises that used body weight, free weights, and cuff weights for resistance with the focus on antigravity muscles and upper-extremity muscles. The 20-30 min training sessions included rest intervals of 30-120 s and an intermediate repetition velocity, and the program went for 12 weeks. Using a handheld dynamometer, the core muscles, antigravity muscles, and the upper-extremity muscles were tested before, during (at 6 weeks), and after intervention (at 12 weeks). The results showed improvement in each muscle tested, with the most substantial gains in strength in the rectus abdominis (300\% strength improvement) and gluteus medius muscles (300-500\% strength improvement). Another case study involving a seven-year-old child demonstrated improvements in performance on all posttest measures following 24 
strength training sessions. Gross-motor delays were not consistent with DCD when measured with BOT-2 and DCD-Q (Menz et al., 2011). Of these two articles, one reported statistical significance (Kaufman \& Schilling, 2007).

Core Stability Training. Three studies used core stability training as the physical therapy intervention for 55 children ages 6-16 years (Au et al., 2014; Bhayani \& Singaravelan, 2012; Kane \& Bell, 2009). The programs ranged from 6 to 8 weeks. One study provided core stability training coupled with task-specific training through group sessions and a home program, and the results showed that each child improved in one or more of the following areas: motor skill, self-efficacy for physical activity, and core stability outcome measures (Kane \& Bell, 2009).

Another study compared core stability training and task-oriented training program by randomly allocating participants to the core stability program or the task-oriented motor program (Au et al., 2014). The short form of the BOT-2 and SOT tests were used at pre- and postintervention, and neither a significant betweengroup difference in the change of motor proficiency standard score $(p=.72)$ nor a significant between-group difference in composite equilibrium score from the SOT $(p=.10)$ was found. However, there were improvements in motor proficiency in both groups (core stability group mean change $(S D)=6.3(5.4) ; p=.01$ and taskoriented group mean change $(S D)=5.1(4.0) ; p=.01)$. The task-oriented group showed improvement in composite equilibrium score (mean change $(S D)=6.0(5.5)$; $p=.01)$; the core stability group did not (mean change $(S D)=0.0(9.6) ; p=.81$ ).

In a third study, participants were randomized into a core stability training, a task-specific physical activity group (Group A) and a task-specific physical activity group (Group B) for 6 weeks (Bhayani \& Singaravelan, 2012). BOT-2 and CSAPPA were both used to assess effectiveness. The improvement in Group A was statistically significant with $p<.05$ and highly significant in Group B with $p$ $<$.01. P-values of the BOT-2 and CSAPPA scores of both groups were each 0.01 . Coordination improved incrementally by $9.20 \%$, balance improved by $8.52 \%$, strength improved by $4.10 \%$, and speed and agility improved by $3.05 \%$ in Group A. These studies showed that core stability programs improve motor proficiency and promote physical activity, improving quality of life in children with DCD (Au et al., 2014; Bhayani \& Singaravelan, 2012; Kane \& Bell, 2009). Of these three studies, two reported mean differences within intervention groups, as seen in Figure 4.

\section{Contemporary Physical Therapy}

Active Virtual Gaming. Since AVG is a relatively new type of intervention, there are few studies on its effect, especially in the DCD population. Larke, Campbell, Jensen, and Straker (2015) compared the responsiveness of the MABC with another tool, Motion Analysis Laboratory (MAL) data, for evaluating AVG intervention effects in children with DCD statistically and meaningfully (Larke et al., 2015). Effect sizes for the MABC and MAL were moderate to large (0.7-1.8). MABC detected a larger percentage of children with a high degree of change (47.6-71.4\%) than MAL (0-19.0\%) (Larke et al., 2015).

Gonsalves et al. (2015) used the MAL in a quasi-experimental study to compare the quality of motor pattern between children with DCD and typically developing (TD) children. Twenty-one children with DCD from ages 10-12, and 19 age- and gender-matched TD children played a match of table tennis on both a 
Sony PlayStation 3 Move and a Microsoft Xbox Kinect. Hand path, wrist angle, and elbow angle were recorded, and the result data showed a difference in motor patterns between the children. Data from the children with DCD reflected slower path speed difference (backhand mean difference $[M D]=1.20 \mathrm{~m} / \mathrm{s}$ ), greater wrist extension (forehand $\mathrm{MD}=34.3$ degrees), and greater elbow flexion (forehand MD $=22.3$ degrees) compared with TD children when engaged in AVG. There were differences in movement patterns used between AVG types (Gonsalves et al., 2015).

Interactive Metronome Training. A new intervention called Interactive Metronome (IM) for improving timing and coordination was tested in a case study (Bartscherer \& Dole, 2005). The IM was used by physical therapists to assess the child's timing and accuracy. The child underwent a seven-week training program with the IM and was assessed before, during, and after training. The child exhibited marked change in his gross and fine motor skills BOT-2 scores on both timing and accuracy and on several BOT-2 subtests.

\section{Hippotherapy}

The effect of the hippotherapy intervention method on children with DCD—specifically those with mild-to-moderate balance problems-was assessed to determine whether there was a correlation between balance and function (Silkwood-Sherer et al., 2012). One repeated-measures study included 16 participants between the ages of 9-16 years. Intervention consisted of 45 min hippotherapy sessions twice per week for 6 weeks. Two baseline assessments and one post intervention assessment of balance were measured using the PBS and ASKp, both tools were found to be statistically significant for all measurements ( $p<.00$ for both measures). A statistical difference was found between baseline and post intervention measures $(p<.01)$ which resulted in large effect sizes for PBS $(d=1.6)$ and ASKp $(d=1.5)$ scores after hippotherapy (Silkwood-Sherer et al., 2012).

\section{Aquatic and Rebound Therapy}

Other interventions can be explored individually or combined as a comprehensive method of treatment. While Hillier, McIntyre, and Plummer (2010) investigated the use of aquatic therapy alone in a randomized controlled trial on improving motor skills in children with DCD, Addy (1996) employed both aquatic and rebound therapy methods involving trampolines to provide therapeutic exercises for the patient in a blinded randomized controlled trial.

In the aquatic therapy randomized controlled trial, 13 children with DCD were randomly allocated to the intervention group, receiving six weekly aquatic therapy sessions for 30 min for 6-8 weeks, or to a wait-list control group (Hillier et al., 2010). The MABC and PSPCSA were the assessment tools used, and an assessor blinded to the participant groups conducted all assessments. Using ANCOVA, mean MABC scores at posttest were higher for children in the intervention group than scores for children on the wait-list $(p=.06)$. Similar trends were noted on the physical competence portion of the PSPCSA $(p=.06)$.

Addy (1996) used a combined intervention of aquatic therapy, rebound therapy, and perceptual/proprioceptive stimulation with an outcome focus of movement, balance, and coordination in water (Addy, 1996). Each of the three 
treatment approaches lasted for 6-7 weeks. Children (age range: 4.6-12 years) were put into three groups, with 8-10 per group. They attended one session each week for one hour. The MABC was used to measure motor performance. Even though statistical data were not reported, the authors reported an improvement in motor performance.

A recent study aimed to investigate the possible effects of a balance training program on children with DCD (Giagazoglou, Sidiropoulou, Mitsiou, Arabatzi, \& Kellis, 2015). Twenty children were split into two groups-each individual of the experimental group was paired with an individual of the control group. Participants were tested at baseline and after the end of a 12-week period by performing static balance control tasks while standing on an EPS pressure and being videotaped. The results showed that the participants improved in motor coordination and balance due to rebound therapy (Giagazoglou et al., 2015). The researchers found no statistically significant TBCT differences between groups or in the control group between measurements for each skill measured.

\section{Task-Oriented Approach: Neuromotor Task Training}

Neuromotor task training was studied in research involving 152 participants ages 5-10 years (Ferguson, Jelsma, Jelsma, \& Smits-Engelsman, 2013; Niemeijer et al., 2007; Niemeijer, Schoemaker, \& Smits-Engelsman, 2006; Niemeijer, SmitsEngelsman, Reynders, \& Schoemaker, 2003; Schoemaker et al., 2003). Three of the studies reported their NTT effect sizes. Figure 5 displays the summation of these studies' findings.

Researchers in one study were blinded to group allocation when assessing results (Niemeijer et al., 2007). In this study, the difference between the MABC pre- and postintervention scores were statistically significant in the intervention group $(p=.003)$, while there was no significant change in scores in the control group. Likewise, the motor patterns of the intervention group improved while the control-group participants' motor patterns worsened $(p<.001)$. Furthermore, older children improved more on the TGMD-2 $(p=.01)$ when adjusted for the children's initial TGMD-2 scores $(p<.001)$. For the MABC, no such association was found $(p=.25)$ (Niemeijer et al., 2007).

In another study, participants were allocated to either a NTT group $(N=$ $27)$ or a Wii training group $(N=19)$ (Ferguson et al., 2013). To assess pre- and postintervention performance, researchers used the MABC, a handheld dynamometer, the Functional Strength Measure, the Muscle Power Sprint Test, and the $20 \mathrm{~m}$ Shuttle Run Test. The NTT program was nine weeks, and the Wii Fit program was six weeks. Measure outcomes were carried out by two different assessors blinded to which subject received or did not receive treatment. The total FSM score improved significantly in the NTT group $(p<.01)$ but did not in the Wii group $(p=.89)$. The anaerobic performance of the NTT group $(p<$ $.00)$ and the Wii group $(p=.01)$ improved significantly; however, the aerobic performance of the Wii group did not improve $(p=.88, d=.02)$, while the NTT group who showed statistically significant improvement and large effect size ( $p$ $=.02, d=-1.1$ ).

A third study using NTT showed significant improvement in handwriting after 18 therapy sessions (Schoemaker et al., 2003). Fifteen children with 


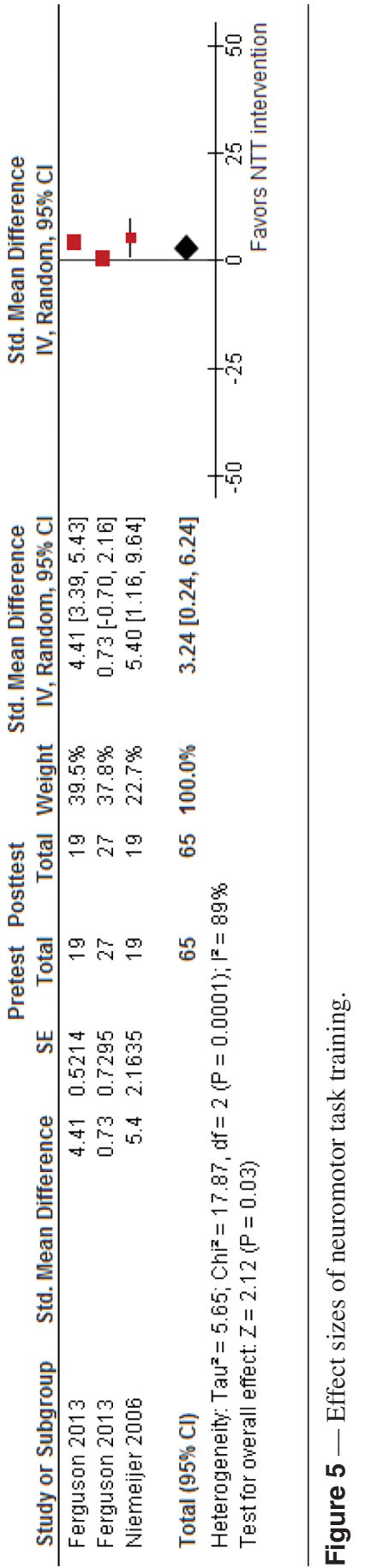


DCD participated in this study. Ten children were included in the intervention group and five children were included in a no-treatment control group. Children in the intervention group participated individually in $30 \mathrm{~min}$ sessions weekly. Testing using the MABC and a dysgraphia scale (BHK) occurred three times-before intervention (T1), after nine sessions (T2), and after another nine sessions (T3) - to measure the progression of gross and fine motor skills and handwriting in particular. Participants in the control group were tested on the MABC at T1 and T2 to measure spontaneous movement. Significant improvement was seen in the intervention group from T1 to T3 $(p=.02)$ and for handwriting quality between T1 and T3 $(p=-.02)$, but not for handwriting speed $(p=.11)$.

The effectiveness of NTT also relies on therapists' use and application of evidence-based teaching principles when providing instructions and feedback (Niemeijer, et al., 2006). Two different studies investigated whether the use of different teaching principles during NTT were associated with treatment effects and also which teaching principles were effective for children with DCD during NTT (Niemeijer et al., 2003; Niemeijer, et al., 2006). First introduced in 2003, Motor Teaching Principles Taxonomy (MTPT) was developed to investigate which teaching principles physical therapists used to treat children with DCD during NTT (Niemeijer et al., 2003). In this study, 23 children with DCD, ages 5-10, and 13 female physical therapists participated. One NTT treatment session was recorded but not until each child completed six sessions. MTPT teaching principles are categorized into three categories: Giving Instructions, Sharing Knowledge, and Providing or Asking for Feedback. The results of this study showed that the reliability and validity of MTPT were satisfactory. Feedback was provided by therapists to a child after a poor performance on TGMD-2 ( $p$ $=.09)$; the same therapists shared knowledge about the task's difficulty after a poor performance on $\operatorname{MABC}(p=.06)$.

The other study found an improvement in the motor performance of children with DCD through instructions including giving task completion clues, asking questions about tasks, and explaining why a movement should be executed in a certain way (Niemeijer et al., 2006). This 2006 study included 19 children with DCD (mean age $=7.5$ years) and involved 11 physical therapists to examine whether the use of different teaching principles during NTT were associated with treatment effects. Weekly 30 min NTT sessions occurred over a nine-week period. Posttest assessments using the MABC and TGMD-2 were completed one or two weeks after the ninth session and found a statistically significant improvement over the pretest scores. More precisely, post hoc analyses of children tested three times showed a statistically significant improvement in the MABC $(p=.02)$ and TGMD-2 $(\mathrm{p}=.05)$ scores between first and second measurement but not between second and third. Researchers videotaped one of the last three sessions with the MTPT observation system that quantified the number of motor teaching principles associated with NTT and the frequency of their use. Numerous instruction methods within the categories of Giving Instruction, Sharing Knowledge, and Providing or Asking for Feedback were effective on improving scores. For example, after nine sessions, Giving Clues was significantly associated with TGMD-2 improvement $(p=.01)$ (Niemeijer et al., 2006). 


\section{DISCUSSION}

The purpose of this study was to conduct a systematic review to gather all existing knowledge on interventions in physical therapy contexts for children with DCD. More specifically, our goals were to identify the types of physical therapy interventions that have been explored in children with DCD and the most common variables being addressed (such as balance, motor coordination and timing, and muscle strength and function), and to find out whether these interventions are effective. After evaluating 3,570 abstracts and 109 full-text papers, we were able to locate 29 primary studies addressing the purpose of this paper, and 13 of these studies were included in the meta-analysis. The types of interventions in PT contexts were categorized into Task-Oriented, Traditional, and Contemporary.

The results of this systematic literature review support the notion that taskoriented approaches and traditional and contemporary physical therapy interventions are effective treatment methods for children with DCD. In addition, the meta-analysis supports the notion that interventions in PT contexts are effective compared with control groups, and children significantly improved the outcome variable on the posttest compared with the pretest. In the studies where occupational therapy was combined with physical therapy, the outcomes of these interventions were not significantly different, possibly due to the sample size (only two studies being compared). Therefore, as we compiled the results of the 29 articles, it is possible to say that PT interventions are effective for overall improvements in children with DCD.

\section{Limiting Factors}

Obviously, the term physical therapy has particular connotations in some (clinical) circles and, as such, might influence whether therapists use a traditional theoretical stance or a more empirically driven model. Even though the metaanalysis showed significance between intervention and control groups, there is high risk of bias, as the majority of articles included were not homogenous and many did not involve random allocation into groups. Some additional potential limiting factors have been identified as reasons for some of the inconsistencies found within the results. These factors include sample size, motivation, duration, and intervention design.

\section{Sample Size}

The major limitation experienced by most of the studies was sample size. The authors of one study commented that even though they found an increase in motor proficiency after the core stability and task-oriented programs, no firm conclusion can be made due to the small sample size (Au et al., 2014). Having a sufficient amount of subjects participating in a study helps reduce the chances of error. Hillier et al. (2010) identified a small sample size as a limiting factor that possibly led to lack of significant findings for some of the variables (confounding variables), while Chia \& Chua (2002) stated that a larger sample size would have resulted in more conclusive results. In future studies, larger sample sizes can better detect causation, amplify acute outcomes, and decrease bias. 


\section{Motivation}

Motivation is a key factor in a research program, especially if the participants are children. The DCD population studied was mostly school-aged children, and when children are motivated, they want to learn and do better, which is usually reflected in the results. For example, Kaufman \& Schilling (2007) changed their assessment tools to motivate their five-year-old subject, by deciding not to do manual muscle testing and using a handheld dynamometer (HHD). They reported that the subject was less frustrated with the HHD, performed better with an increased consistency, which increased his motivation to continue. In order for there to be an increase in motivation, intervention needs to be fun for the children. Rebound therapy intervention by Giagazoglou et al. (2015) stated that motivation played a key role in the subject's participation because they enjoyed the activity; also subjects who took part in the Wii Fit program in the Ferguson et al. (2013) study reported that they enjoyed the program and were motivated to attend sessions. However, on the other end of the spectrum, when the activity was not enjoyable, participants' lack of motivation was seen, such as in the Fong et al. (2013) study.

\section{Duration}

From the reviewed articles, the duration of the intervention ranged from 1 to 36 weeks, and the amount of time per session ranged from 20 min to $2 \mathrm{hr}$. Sufficient duration could lead to an improvement in results. Ferguson et al. (2013) study showed that their Wii Fit group didn't improve in motor performance; researchers partly attributed that result to shorter duration of the program for the Wii group (6 weeks) as compared with the NTT group ( 9 weeks). The authors wondered if there would have been greater improvement in scores of the Wii group if there had been more time. Hillier et al. (2010) stated that due to limits in resources, time, and subjects, the study was carried out pragmatically, and this pragmatic approach allowed a greater risk of bias or inconclusive findings. Longer duration means more practice, and more practice means an increased chance of retaining information and storing it in the long-term memory. Information in the long-term memory can be stored for long periods of time. This is beneficial for children with DCD because not only do they show improvement in motor skills, but they retain it for a long time.

\section{Intervention Design}

The design of an intervention is essential to the success of a study. Usually, a randomized controlled trial (RCT) with blind assessment is an ideal experimental research design; however, the design is dependent on the purpose of the research. For example, in the Giagazoglou et al. (2015) study, a matched-pair experimental design was used; however, the authors stated that an RCT would have been better and would have allowed for safer conclusions regarding efficacy of the experimental design. If the purpose of the study is to find the effectiveness of a program, it is always best to have an experimental group (testing the program) and a control group to compare. This was not the case for the Hung \& Pang (2010) and the Peters \& Wright (1999) studies. Fong et al. (2013) used an intention-to-treat principle but stated that it may have contributed to the "no change" observations in their 2 control groups. In the Hillier et al. (2010) study, an assessor blinded to the participant 
group conducted all assessments, but the subjects and the treating therapists were not blinded, which, according to authors, introduced a source of potential bias.

\section{Implications for Future Research}

Based on the discussion above, it can be concluded that future studies need to include activities that are therapeutic yet enjoyable for increased motivation, larger sample size, sufficient duration, and the right study design. It is also recommended that further research on interventions in PT contexts for children with DCD include a follow-up. A follow-up is beneficial for assessing long-term effects of the intervention. From the 29 included studies, only three conducted a follow-up on subjects (Iversen et al., 2005; Lee \& Smith, 1998; Morton, 2015). The follow-up in Iversen et al. (2005) revealed that the subjects still practiced and enjoyed the activities 1-4 years later with positive health and social implications. The duration between postintervention and follow-up should be sufficient enough for a change to be seen when assessed. Niemeijer et al. (2006) suggested a follow-up to assess motor skill retention over a long period of time.

\section{Recommendations for Practice}

In 2006, D.A. Sugden developed the Leeds Consensus Statement, which highlighted the term developmental coordination disorder and recognized the disorder as distinct and unique, outlining recommendations for the application of diagnostic criteria. The Consensus guidelines for intervention approaches (which can be applied to physical therapy interventions) are as follows: interventions should "contain activities that are functional and are based on those relevant to daily living and meaningful to the child, parents, teachers, and others", and should also take account of the wishes of the child, the significant persons in the child's life, and the child's contextual life and family circumstances while being theory- and evidence-based (Sugden, 2006, p. 6). Hadders-Algra (2000) stated that "an intervention should provide active practice in the skills that are deficient to enhance the right selection of neuronal groups that will lead to an increment of adaptation of motor behavior" (p. 162). Physical therapists should adopt this principle of repetition in their intervention approach to accommodate for adaptation of the skills administered to the child. More practice also means prescribing home exercises to the child in addition to the intervention.

Giagazoglou et al. (2015) recommended that "interventions for children with DCD should focus on sensorimotor organization and include activities organizing the sensory system by providing visual, proprioceptive, auditory, and tactile inputs" (p. 18).

\section{CONCLUSION}

Although more research for interventions in physical therapy contexts and DCD needs investigating, the research that has been carried out under this topic shows that physical therapy intervention is an effective treatment for children diagnosed with DCD, especially for improving balance, motor coordination and timing, muscle strength and function, and motor function/performance. Based on the articles 
reviewed, we can conclude that task-oriented approaches (NTT) and motor training-based interventions of traditional and contemporary PT contexts are beneficial for improving outcomes of children with DCD. Interventions based on motor skills training and NTT are best for gross motor problems. Additionally, NTT is a great approach for improving fine motor problems. Based on that, future studies should continue exploring the effectiveness of these therapy modalities and the specific outcomes in children with DCD.

\section{Acknowledgments}

The authors would like to thank Helen Mayo for peer-reviewing the search strategy.

\section{References}

Addy, L.M. (1996). A multitprofessional approach to the treatment of developmental coordination disorder. British Journal of Therapy and Rehabilitation, 3(11), 593-599. doi:10.12968/bjtr.1996.3.11.14738

American Physical Therapy Association. (2015). Who are physical therapists? Retrieved from http://www.apta.org/AboutPTs/

American Psychiatric Association. (2013). Neurodevelopmental disorders. In Diagnostic and Statistical Manual of Mental Disorders (5th ed.). doi:10.1176/appi. books.9780890425596.dsm01

Au, M.K., Chan, W.M., Lee, L., Chen, T.M.K., Chau, R.M.W., \& Pang, M.Y.C. (2014). Core stability exercise is as effective as task-oriented motor training in improving motor proficiency in children with developmental coordination disorder: A randomized controlled pilot study. Clinical Rehabilitation, 28(10), 992-1003. doi:10.1177/0269215514527596

Bartscherer, M.L., \& Dole, R.L. (2005). Interactive Metronome training for a 9-year-old boy with attention and motor coordination difficulties. Physiotherapy Theory and Practice, 21(4), 257-269. doi:10.1080/09593980500321085

Bhayani, K., \& Singaravelan, R.M. (2012). Effectiveness of core stability training programme on improving task specific physical activity in developmental coordination disorder children. Revista Românã De Kinetoterapie, 18(30), 33-40.

Camden, C., Rivard, L., Pollock, N., \& Missiuna, C. (2012). Physiotherapists can make a difference in the lives of children with Developmental Coordination Disorder (DCD). Retrieved from http://elearning.canchild.ca/dcd_pt_workshop/index.html

Cermark, S.A., Gubbay, S.S., \& Larkin, D. (2009). What is developmental coordination disorder? In S.A. Cermak \& D. Larkin (Eds.), Developmental Coordination Disorder (pp. 2-22). Albany, NY: Delmar Thompson Learning.

Chia, L.C., \& Chua, L.W. (2002). Effects of physiotherapy on school-aged children with DCD and learning difficulties: A pilot study. Physiotherapy Singapore, 5(4), 75-80.

Ferguson, G.D., Jelsma, D., Jelsma, J., \& Smits-Engelsman, B.C.M. (2013). The efficacy of two task-orientated interventions for children with developmental coordination disorder: Neuromotor task training and Nintendo Wii Fit training. Research in Developmental Abilities, 34, 2449-2461. doi:10.1016/j.ridd.2013.05.007

Fong, S.S.M., Chung, J.W.Y., Chow, L.P.Y., Ma, A.W.W., \& Tsang, W.W.N. (2013). Differential effect of Taekwondo training on knee muscle strength and reactive and static balance control in children with developmental coordination disorder: A randomized controlled trial. Research in Developmental Abilities, 34, 1446-1455. doi:10.1016/j. ridd.2013.01.025

Giagazoglou, P., Sidiropoulou, M., Mitsiou, M., Arabatzi, F., \& Kellis, E. (2015). Can balance trampoline training promote motor coordination and balance performance 
in children with developmental coordination disorder? Research in Developmental Abilities, 36, 10-19.

Gonsalves, L., Campbell, A., Jensen, L., \& Straker, L. (2015). Children with developmental coordination disorder play active virtual reality games differently than children with typical development. Physical Therapy, 95(3), 360-368.

Hadders-Algra, M. (2000). The neuronal group selection theory: Promising principles for understanding and treating developmental motor disorder. Developmental Medicine and Child Neurology, 42, 707-715.

Hee, K., \& Wilson, C. (2008). Effects of physical therapy in children with developmental coordination disorder (Unpublished doctoral dissertation). Pacific University Oregon College of Health Professions, Hillsboro, OR. Retrieved from http://commons.pacificu. $\mathrm{edu} / \mathrm{pt} / 42 /$

Hillier, S. (2007). Intervention for children with developmental coordination disorder: A systematic review. Internet Journal of Allied Health Sciences and Practice, 5(3), 1-11.

Hillier, S., McIntyre, A., \& Plummer, L. (2010). Aquatic physical therapy for children with developmental coordination disorder: A randomized controlled trial. Physical \& Occupational Therapy in Pediatrics, 30(2), 111-124. doi:10.3109/01942630903543575

Hodges, M.L. (2014). Applying developmental coordination disorder treatment theories to physical therapy practice: A case report (Unpublished doctoral dissertation). Fort Myers, FL: Florida Gulf Coast University College of Health Professions and Social Work. Retrieved from http://fgcu.digital.flvc.org/islandora/object/fgcu\%3A27281/ datastream/OBJ/view/Applying_developmental_coordination_disorder_treatment_ theories_to_physical_therapy_practice_a_case_report_.pdf.

Hung, W.W.Y., \& Pang, M.Y.C. (2010). Effects of group-based versus individual-based exercise training on motor performance in children with developmental coordination disorder: A randomized controlled pilot study. Journal of Rehabilitation Medicine, 42, 122-128. doi:10.2340/16501977-0496

Iversen, S., Ellertsen, B., Tytlandsvik, A., \& Nødland, M. (2005). Intervention for 6-year-old children with motor coordination difficulties: Parental perspectives at follow-up in middle childhood. Advances in Physiotherapy, 7(2), 67-76. doi:10.1080/14038190510010304

Kane, K., \& Bell, A. (2009). A core stability group program for children with developmental coordination disorder: 3 clinical case reports. Pediatric Physical Therapy, 21, 375-382. doi:10.1097/PEP.0b013e3181beff38

Kaufman, L.B., \& Schilling, D.L. (2007). Implementation of a strength training program for a 5-year-old child with poor body awareness and developmental coordination disorder. Physical Therapy, 87(4), 455-467. doi:10.2522/ptj.20060170

Larke, D., Campbell, A., Jensen, L., \& Straker, L. (2015). Responsiveness of clinical and laboratory measures to intervention effects in children with developmental coordination disorder. Pediatric Physical Therapy, 27, 44-51. doi:10.1097/PEP.0000000000000102

Lee, M.G., \& Smith, G.N. (1998). The effectiveness of physiotherapy for dyspraxia. Physiotherapy, 84(6), 276-284. doi:10.1016/S0031-9406(05)65529-0

Maldonado, G., Miller, D.P., Moran, T., \& Eberhart, P. (2012). Retention of treatment related to motor performance for children diagnosed with DCD: A systematic review. Pediatric Physical Therapy, 96-128. Pediatric Section Poster Presentation, APTA Combined Sections Meeting.

Menz, S., Hatten, K., \& Beuttler, M. (2011). Effectiveness of a strength-training intervention for a 7-year-old girl with gross motor delays consistent with developmental coordination disorder: A case study. Pediatric Physical Therapy, 125-126. Abstracts of Poster and Platform Presentations, Combined Sections Meeting.

Miller, L.T., Polatajko, H.J., Missiuna, C., Mandich, A.D., \& Macnab, J.J. (2001). A pilot trial of a cognitive treatment for children with developmental coordination disorder. Human Movement Science, 20(1-2), 183-210. 
Morton, C. (2015). The effect of a group motor skills programme on the participation and movement ability of children with Developmental Coordination Disorder. ProQuest Dissertations \& Theses, 1-144.

National Institutes of Health. (2014). Quality assessment tool for before-after (pre-post) studies with no control group [Measurement instrument]. Retrieved from https:// www.nhlbi.nih.gov/health-pro/guidelines/in-develop/cardiovascular-risk-reduction/ tools/before-after

Niemeijer, A.S., Schoemaker, M.M., \& Smits-Engelsman, B.C.M. (2006). Are teaching principles associated with improved motor performance in children with developmental coordination disorder? A pilot study. Physical Therapy, 86(9), 1221-1230. doi:10.2522/ ptj.20050158

Niemeijer, A.S., Smits-Engelsman, B.C.M., Reynders, K., \& Schoemaker, M.M. (2003). Verbal actions of physiotherapists to enhance motor learning in children with developmental coordination disorder. Human Movement Science, 22, 567-581. doi:10.1016/j. humov.2003.09.010

Niemeijer, A.S., Smits-Engelsman, B.C.M., \& Schoemaker, M.M. (2007). Neuromotor Task Training for children with developmental coordination disorder: A controlled trial. Developmental Medicine and Child Neurology, 49, 406-411. doi:10.1111/j.14698749.2007.00406.x

Physiotherapy Evidence Database. (1999). PEDro scale [Measurement instrument]. Retrieved from http://www.pedro.org.au/english/downloads/pedro-scale

Peters, J.M., \& Wright, A.M. (1999). Development and evaluation of a group physical activity programme for children with developmental co-ordination disorder: An interdisciplinary approach. Physiotherapy Theory and Practice, 15, 203-216. doi:10.1080/095939899307621

Schoemaker, M.M., Hijlkema, M.G.J., \& Kalverboer, A.F. (1994). Physiotherapy for clumsy children: An evaluation study. Developmental Medicine and Child Neurology, 36, 143-155. doi:10.1111/j.1469-8749.1994.tb11823.x

Schoemaker, M.M., Niemeijer, A.S., Reynders, K., \& Smits-Engelsman, B.C.M. (2003). Effectiveness of neuromotor task training for children with developmental coordination disorder: A pilot study. Neural Plasticity, 10(1-2), 155-163. doi:10.1155/NP.2003.155

Shea, B.J., Grimshaw, J.M., Wells, G.A., Boers, M., Andersson, N., Hamel, C., Bouter, L. M. (2007). Development of AMSTAR: A measurement tool to assess the methodological quality of systematic reviews. BMC Medical Research Methodology, 7, 10.

Silkwood-Sherer, D.J., Killian, C.B., Long, T.M., \& Martin, K.S. (2012). Hippotherapy-an intervention to habilitate balance deficits in children with movement disorders: A clinical trial. Physical Therapy, 92(5), 707-717.

Smits-Engelsman, B.C.M., Blank, R., Van Der Kaay, A.C., Van Der Meijs, R.M., Vlugt-Van Den Brand, E., Polatajko, H.J., \& Wilson, P.H. (2013). Efficacy of interventions to improve motor performance in children with developmental coordination disorder: A combined systematic review and meta-analysis. Developmental Medicine and Child Neurology, 55, 229-237. doi:10.1111/dmcn.12008

Sugden, D.A. (Ed.). (2006). Leeds consensus statement: Development coordination disorder as a specific learning difficulty. Leeds, UK: DCD-UK Dyscovery Centre. Retrieved from http://www.pearsonclinical.co.uk/Psychology/ChildCognitionNeuropsychologyandLanguage/ChildPerceptionandVisuomotorAbilities/MABC-2/Resources/LeedsConsensus06.pdf.

Watter, P., \& Bullock, M.I. (1989). A physiotherapy directed school-based group management programme for children with mild motor and coordination problems. New Zealand Journal of Physiotherapy, 17(2), 19-23. 


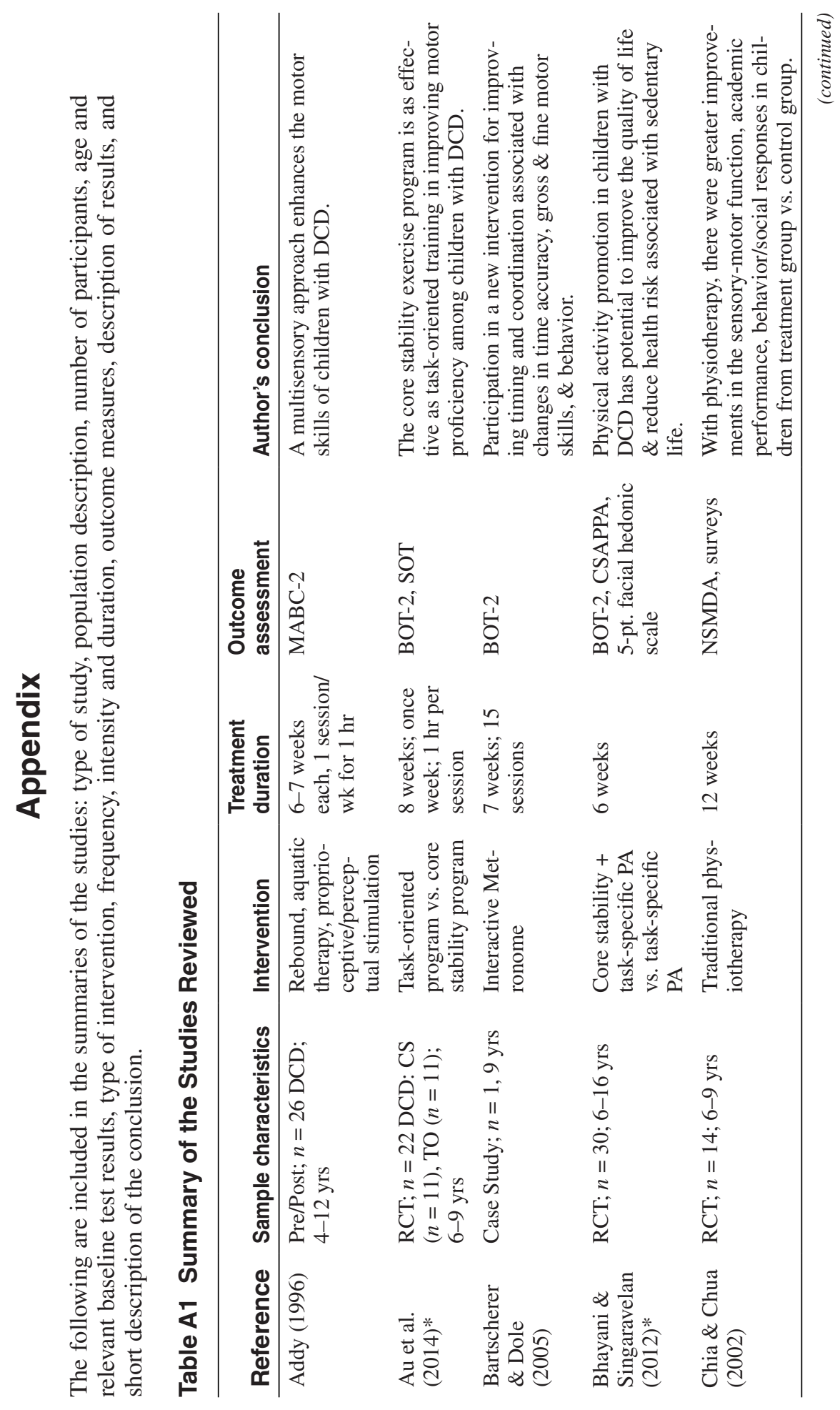




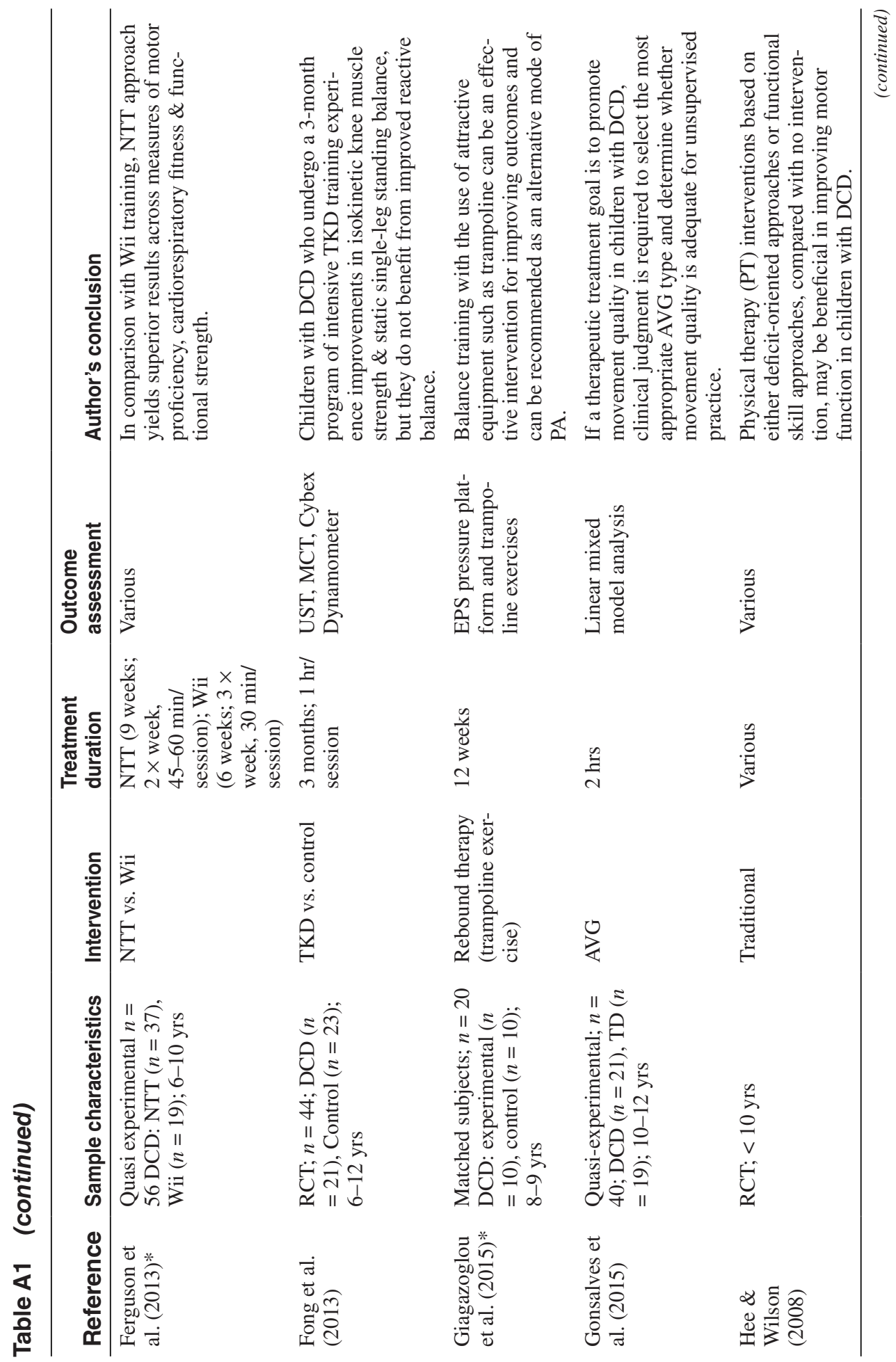




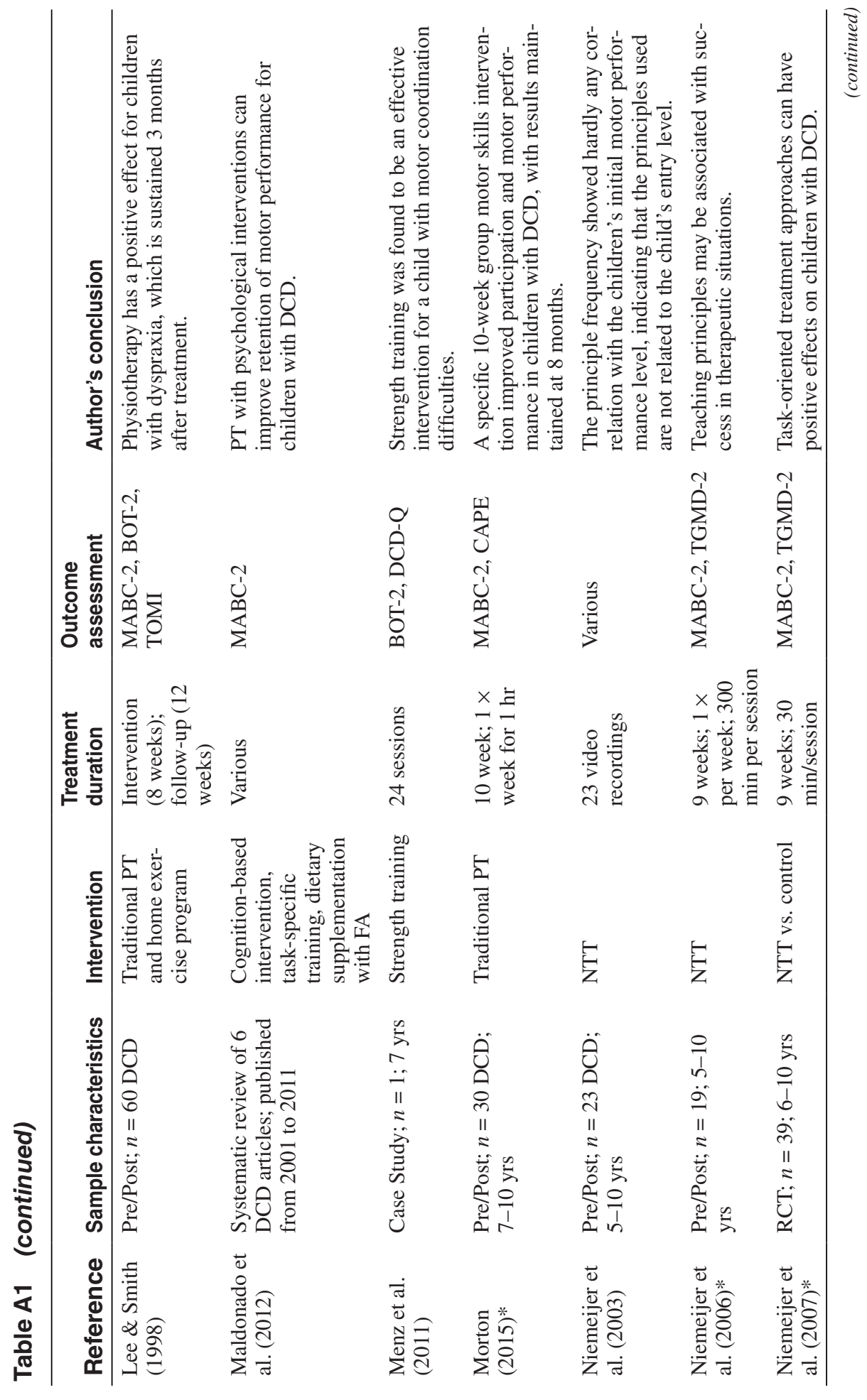




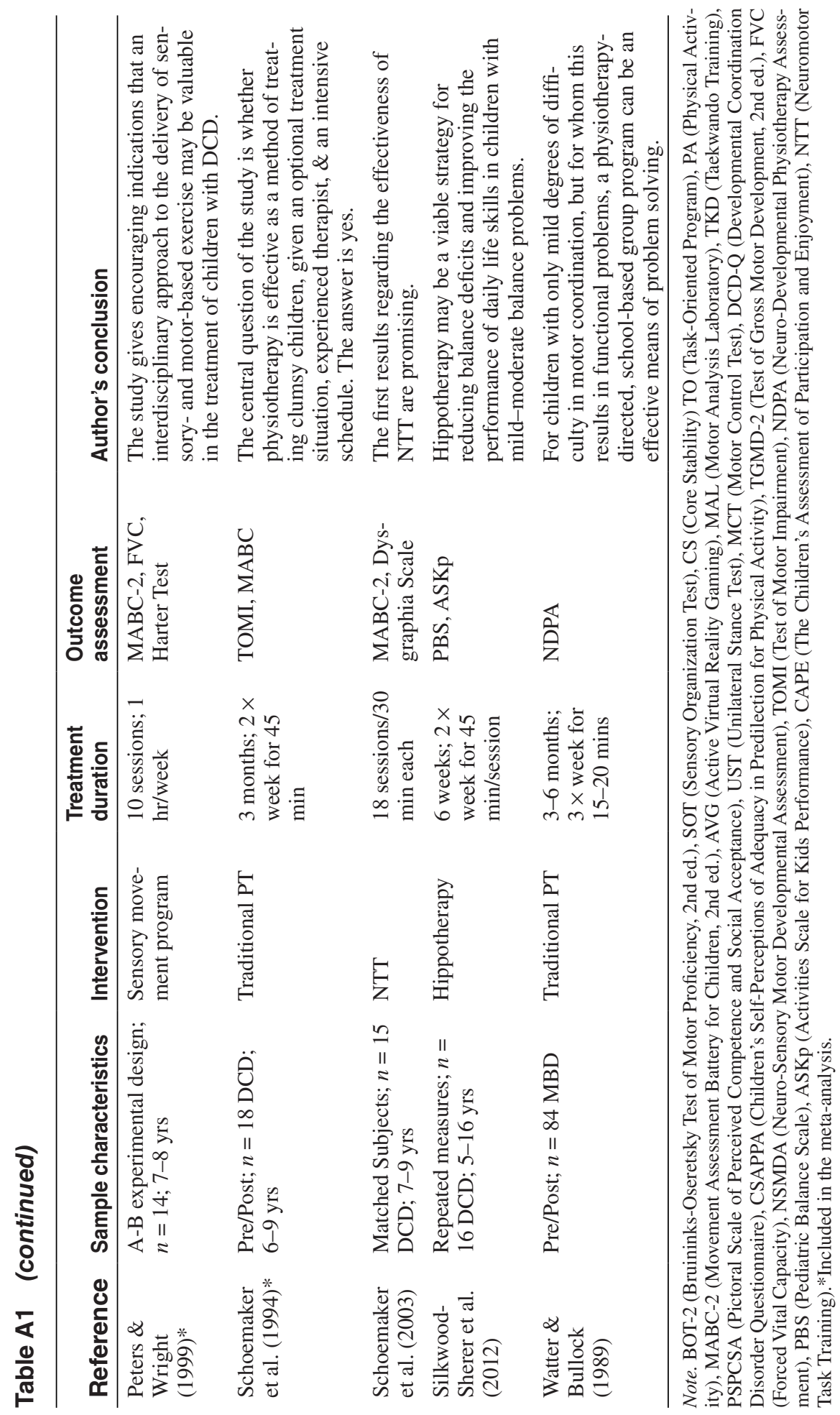

